\title{
Cellular Models for the Study of Prions
}

\author{
Brandon B. Holmes ${ }^{1,2}$ and Marc I. Diamond ${ }^{2}$ \\ ${ }^{1}$ Medical Scientist Training Program, Washington University School of Medicine, St. Louis, Missouri 63110 \\ ${ }^{2}$ Center for Alzheimer's and Neurodegenerative Diseases, University of Texas Southwestern Medical Center, \\ Dallas, Texas 75390 \\ Correspondence: marc.diamond@utsouthwestern.edu
}

\begin{abstract}
It is now established that numerous amyloid proteins associated with neurodegenerative diseases, including tau and $\alpha$-synuclein, have essential characteristics of prions, including the ability to create transmissible cellular pathology in vivo. We have developed cellular bioassays that report on the various features of prion activity using genetic engineering and quantitative fluorescence-based detection systems. We have exploited these biosensors to measure the binding and uptake of tau seeds into cells in culture and to quantify seeding activity in brain samples. These cell models have also been used to propagate tau prion strains indefinitely in culture. In this review, we illustrate the utility of cellular biosensors to gain mechanistic insight into prion transmission and to study neurodegenerative diseases in a reductionist fashion.
\end{abstract}

$P^{r}$ ion detection and characterization has previously depended primarily on serial inoculation of rodent models with infectious $\operatorname{PrP}^{\mathrm{Sc}}$. Such inoculation paradigms can detect minute quantities of infectious $\mathrm{PrP}^{\mathrm{Sc}}$ seeds. In the last 40 years, these animal bioassays have yielded tremendous insights into prion biology, however, they require dozens of animals, months of passage time, and great cost (Prusiner 1998). During the past decade, with the discovery of multiple proteins that have fundamental prion characteristics, the field has shifted to include cellular bioassays that permit quantitative amplification of prions.

Our laboratory has pioneered a set of fluorescence-based cellular biosensors that exquisitely report various stages of the tau and $\alpha$-synuclein transcellular propagation cycle: binding, uptake, and intracellular seeding. Using a combination of microplate fluorescence detection, automated imaging microscopy, and flow cytometry, we have defined several key mechanisms of tau seeding. For example, tau seeds bind heparan sulfate proteoglycans to enter cells via macropinocytosis and propagate aggregation from the outside to the inside of the cell (Holmes et al. 2013). Furthermore, we have defined tau trimers as the minimal unit that propagates infection into the cells (Mirbaha et al. 2015). Additionally, we have discovered that tau acts as a bona fide prion, propagating discrete conformational states, or strains, which can each trigger unique neuropathology in mice via cell-to-cell transfer. We have concluded that strain diversity may underlie the various tauopathy phenotypes (Sanders et al.

Editor: Stanley B. Prusiner

Additional Perspectives on Prion Diseases available at www.perspectivesinmedicine.org

Copyright (C) 2017 Cold Spring Harbor Laboratory Press; all rights reserved; doi: 10.1101/cshperspect.a024026

Cite this article as Cold Spring Harb Perspect Med 2017;7:a024026 
2014; Kaufman et al. in press). Finally, cellbased assays have enabled the detection of tau seeding activity far in advance of other metrics of neurodegeneration, consistent with the idea that seeds drive disease progression (Holmes et al. 2014).

Cell-based reporter systems confer several advantages over animal models. First, they have rapid experimental turnaround time (days) while simultaneously allowing for indefinite propagation of unique prion strains ( $>6$ months). Second, by using specific cell models, each step in the propagation cascade (binding, uptake, seeding, and strain maintenance) can be separately studied to define discrete mechanisms. Finally, the assays facilitate high-throughput approaches for chemical and genetic screening. We will review advances in the cell biology of prion disease with special emphasis on the role of cellular bioassays. These methods promise to help uncover key mechanisms in transcellular propagation of proteopathic seeds and may ultimately facilitate presymptomatic diagnosis of neurodegenerative diseases (Fig. 1).

\section{CELLULAR INTERNALIZATION OF PROTEOPATHIC TAU SEEDS}

In 2009, we created the first cell culture model to study how tau seeds propagate an aggregated state from the outside to the inside of a cell (Frost et al. 2009a). We observed uptake of tau fibrils, but not monomer, into cells via fluid phase endocytosis, induction of fibril formation of intracellular tau, and subsequent transfer of newly aggregated tau between cells. In conjunction with this model, animal studies indicated that inoculation of tau aggregate seeds would initiate intracellular pathology in mouse brain leading to transcellular movement of aggregates (Clavaguera et al. 2009, 2016). These observations first implicated prion mechanisms in tau pathology. In subsequent years, we extended this work to systematically evaluate tau seed internalization using a variety of standardized and automated platforms. This led to the discovery of the cell-surface receptor for tau and synuclein proteopathic seeds - heparan sulfate proteoglycans (HSPGs) (Holmes et al. 2013).

To assay binding and uptake, we created recombinant tau seeds, covalently labeled them with Alexa Fluor dyes (via succinimidyl esteramine reaction), and applied them to media of cultured cells. The fluorescence signal correlates with the total tau bound or internalized by the cell. To assess binding, we incubated cells with labeled tau seeds at $4^{\circ} \mathrm{C}$ to block ATP-driven cellular internalization, without affecting binding to cell-surface receptors. In contrast, when we incubated cells at $37^{\circ} \mathrm{C}$, tau seeds triggered their own internalization into the cell. Treatment of cells with a protease digested extracellular tau, leaving only intracellular tau for detection. We found that it is important to establish parameters for sufficient proteolysis of extracellular tau because different proteopathic seeds (and even different preparations of the same protein) may have unique degradation profiles. Together, these two assays indicated the relative avidity of proteopathic seeds for the cell surface and their activity in inducing cellular internalization.

Confocal microscopy and western blotting have been the standard detection methods used to probe seed:cell interactions and uptake (Horonchik et al. 2005; Lee et al. 2008; Frost et al. 2009a). To establish a more uniform and highthroughput platform, we used automated microscopy and high content analysis (HCA) in HEK293 cells. Automated microscopy allows simultaneous analysis of multiple parameters via fluorescent labels. Data are extracted from single cells, and imaging of thousands of cells enables statistically robust assessments. Automated imaging can provide information on the complex temporal and spatial sequence of molecular events in the cell. For example, in the cell uptake assays, we have deconstructed specific steps with quantitative metrics, including the percentage of cells positive for tau aggregates, the number of aggregates per cell, the average size, and the percentage localized to specific subcellular compartments (i.e., nuclei, lysosomes, and Golgi apparatus). These assays are amenable to miniaturization and can be conducted in 96- or even 384-well plates to 
A
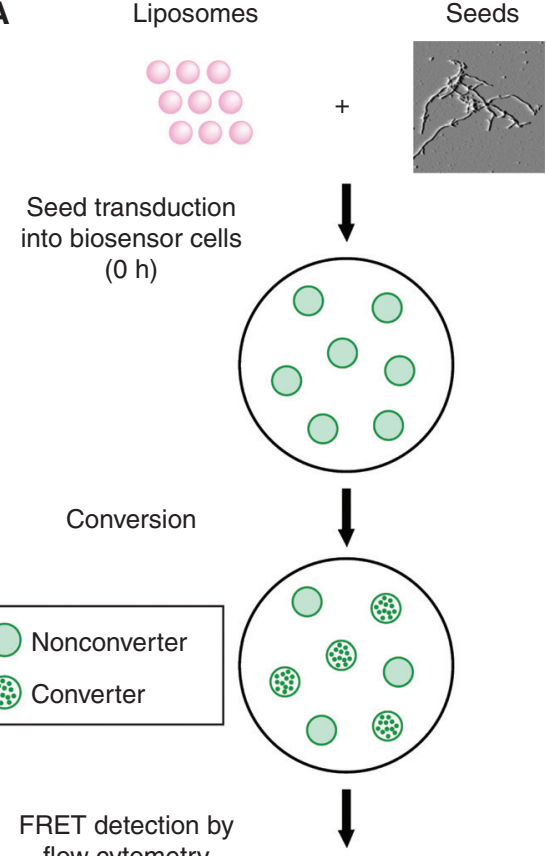
flow cytometry

$(24 \mathrm{~h})$

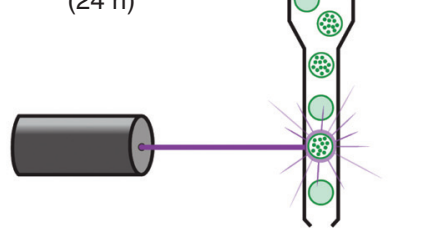

FRET negative : FRET positive

Studying Prion Biology in Cultured Cells

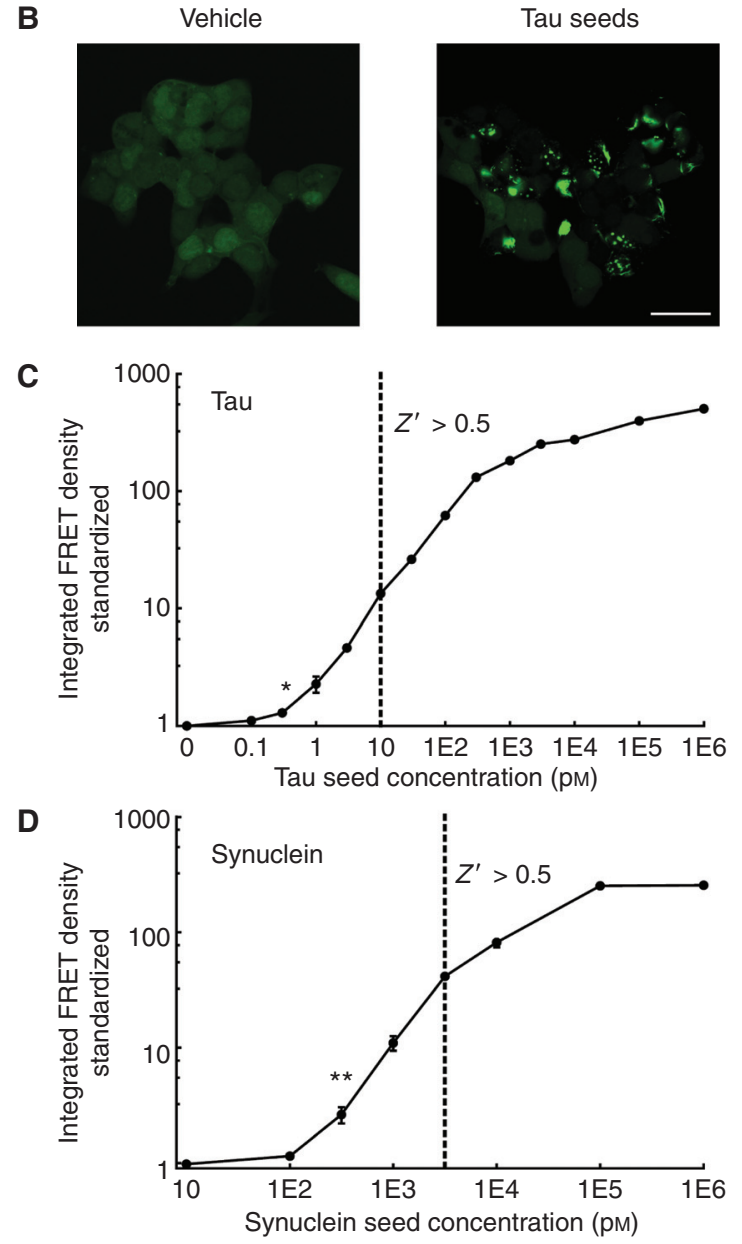

Figure 1. Method to monitor proteopathic seeding activity. $(A)$ Biosensor cells are exposed to proteopathic seeds that have been mixed with lipid-based transfection reagent. After a defined period of time, internalized seeds trigger catastrophic intracellular aggregation of biosensor proteins that are fused to cyan and yellow fluorescent proteins (CFP and YFP). Quenching of CFP by YFP produces fluorescence resonance energy transfer (FRET) that is detected and quantified by flow cytometry. $(B)$ Representative image of cells expressing tau-CFP/YFP treated with vehicle control or tau seeds. Note the induction of intracellular aggregation. $(C)$ Quantification of tau seeding activity over a range of concentrations indicates a high sensitivity to recombinant tau seeds $(0.3 \mathrm{pm}$ monomer equivalent). Note the dynamic range of signal over three logs. (D) Quantification of $\alpha$-synuclein seeding activity based on exposure of biosensor cells expressing $\alpha$-synuclein-CFP/YFP fusion proteins to a range of concentrations of recombinant $\alpha$-synuclein seeds. This assay is less sensitive (300 pM $\alpha$-synuclein monomer equivalent).

investigate many experimental conditions simultaneously. Accurate visualization and quantitation require several stains-a nuclear or DNA stain for $z$-axis focusing and cell counting, and a membrane stain for cell segmentation. We successfully used 4',6-diamidino-2-phenylindole (DAPI) and fluorescently tagged wheat germ agglutinin for these purposes (Holmes et al. 2013).

We combined automated microscopy with confocal and electron microscopy to define the cellular internalization pathway for tau seeds. We found that cells actively take them up via macropinocytosis, a form of fluid-phase bulk 
endocytosis. Tau multimers stimulate their own uptake by binding to HSPGs, glycoproteins that coat the cell surface and serve as ligand binding sites. A 96-well plate format allowed us to titrate HSPG inhibitors to derive precise $\mathrm{IC}_{50} \mathrm{~s}$ and to compare tau binding to that of another HSPGdependent proteopathic seed, $\alpha$-synuclein. We used this platform to identify a novel heparin analog, F6, that blocks uptake of tau seeds in the mouse neocortex (Holmes et al. 2013).

In subsequent studies, our laboratory used similar techniques to elucidate the mechanisms of action of three therapeutic antibodies. We found that microglia have at least two modes of tau seed internalization: an HSPG-dependent mechanism and an antibody/Fc receptor-based mechanism. Anti-tau antibodies can enhance the clearance of tau aggregates through Fc-mediated microglial degradation or inhibit the uptake of tau seeds into neurons, suggesting multiple mechanisms by which antibodies may be protective in tauopathies (Funk et al. 2015). These assays may help to determine the precise epitopes that drive an antibody's response toward a particular clearance pathway, to clarify mechanisms of action in passive immunotherapy, and possibly to predict the behavior of novel antibodies in preclinical trials (Yanamandra et al. 2013).

In another investigation, we exploited these cell-based assays to determine the critical size of tau seeds required for cell uptake. We used size exclusion chromatography to isolate stable tau assemblies of various sizes, ranging from monomer to $>40$ mer. We labeled the assemblies with fluorescent dye and applied them to cells in the binding and uptake assays. All assemblies bound to HSPGs on the cell surface and were sensitive to HSPG inhibitors. However, only tau assemblies of trimer and larger spontaneously triggered cell uptake, indicating that a minimum tau seed size is required to initiate macropinocytosis (Mirbaha et al. 2015). Importantly, Alzheimer's disease (AD)-derived tau trimer also was a minimal unit for infectivity (both in HEK293 cells and in mouse primary neurons), revealing that trimer may serve as the smallest functional assembly for proteopathic seeding activity, and corroborating studies from $>25$ years ago that used radiation-induced inactivation to reach the same conclusion about $\operatorname{PrP}^{\mathrm{Sc}}$ (Bellinger-Kawahara et al. 1988; Mirbaha et al. 2015).

Other laboratories have exploited primary cultured neurons to monitor tau seed trafficking. By using microfluidic chambers (Taylor et al. 2005) Wu et al. (2012) directly exposed somal versus axonal compartments to labeled tau seeds. Both the soma and axons mediated tau aggregate uptake, and after 12 hours of treatment, aggregates had moved throughout the microfluidic compartments. This finding indicated anterograde and retrograde transport of tau seeds in neurons ( $\mathrm{Wu}$ et al. 2012). Most recently, investigators have explored these dynamics using live-cell imaging techniques and HCA to monitor multiple cellular changes after treatment with tau seeds (Usenovic et al. 2015). Automated imaging techniques have, thus, greatly increased our understanding of tau seed internalization and will continue to help define novel molecular targets and pathways.

\section{PROTEOPATHIC SEEDING}

Once internalized, proteopathic seeds act as templates to trap monomer into an assembly whose conformation is determined by the seed itself. This seeded polymerization amplifies the aggregated state. Our group and others have developed cellular tools to detect and quantify proteopathic seeding. We first used fluorescence resonance energy transfer (FRET) to survey tau aggregation states in HEK293 cells, building on previous experience with the huntingtin protein (Pollitt et al. 2003; Kfoury et al. 2012). We genetically fused the tau repeat domain harboring the P301S mutation to cyan fluorescent protein (CFP) and yellow fluorescent protein (YFP) to create a stable monoclonal tau biosensor cell line. In the resting state, the tau fusion proteins are monomeric and FRET-negative. When tau is induced to aggregate by the exogenous application of tau seeds, however, the close approximation of the fluorescent proteins yields FRET on appropriate excitation. Thus, FRET reliably quantifies the seeding activity of exogenous aggregates. Tau biosensor cells are exquisitely 
stable, with no spontaneous aggregation observed after months in culture. Thus, they accurately detect even minor increases in aggregation. Addition of a transfection reagent, such as lipofectamine to the tau seeds before their application to the cells, increases the sensitivity of the assay by as much as 100-fold, because it bypasses the standard cellular uptake mechanism and directly delivers seeds to the cell interior. Using flow cytometry, the biosensor cells measure tau seeds at femtomolar (monomer equivalent) levels and precisely quantify their activity over six log orders of concentration. Importantly, the biosensors respond specifically to tau seeds because heterologous amyloids, including $\alpha$-synuclein and huntingtin, do not induce tau aggregation (Holmes et al. 2014).

We used this assay to profile the temporal evolution of tau seeding activity in the brains of P301S transgenic mice, a model of human tauopathy, and compared it with standard histopathological assessments in the same brains. We hypothesized that seeding would play a proximal role in the development of pathology. We predicted that we would detect seeding activity in the brains of tauopathy mouse models and human subjects before obvious neuropathology, and that this finding would correlate with disease progression. We found that tau seeding activity indeed marks incipient tauopathy, arising as early as 45 days, whereas we could not detect neuropathological changes until at least 120 days using conventional markers of tau pathology, such as MC1, AT8, PG5, and thioflavin S. Thus, tau seeding activity appears very early in neurodegeneration, consistent with a critical upstream role for proteopathic seeds (Holmes et al. 2014). Seeding activity could also be an excellent disease outcome measure for preclinical drug studies, allowing evaluation of drug intervention at very early ages, possibly as early as 45 days. A recent study used the FRET biosensor assay to validate the efficacy of antisense oligonucleotides (ASOs) in an animal model of tauopathy. Tau reduction via ASOs ameliorated degenerative phenotypes and substantially reversed tau seeding activity from P301S mouse brain lysates (SL DeVos, RL Miller, and KM Schoch, submitted).
We have also extended seed-detection assays to human brain. The FRET biosensor cells sensitively detect activity in AD brain homogenates but not in Huntington's disease or nondiseased control brains (Holmes et al. 2014). Future studies will define the temporal evolution of tau seeding activity in $\mathrm{AD}$ brain in relation to the Braak stages of tau pathology. It will be critical to determine whether tau seeding activity precedes classical histopathology in AD. We anticipate that analysis of tau seeding activity (and that of other amyloid proteins) could play a useful role in neuropathological studies.

FRET-based biosensors need not be restricted to flow cytometry (Chun and Johnson 2007; Kfoury et al. 2012). Kaminski Schierle et al. (2011) used the intrinsic fluorescence of amyloids coupled with FRET quenching of a chemical tag to monitor the dynamics of tau aggregation in cells. The $\beta$-sheet structure within amyloids, such as tau and $\alpha$-synuclein produces an intrinsic fluorescence signal. This fluorescence has an insufficient quantum yield for direct in vivo imaging, but when coupled to a FRET donor, such as an Alexa Fluor dye, the amyloid quenches its emission, reducing fluorescence lifetime proportionate to fibril growth (Kaminski Schierle et al. 2011). Using this technology, Michel et al. (2013) concluded that tau accumulates within endosomal compartments before exiting the cell in an aggregated form. If coupled to ultraresolution imaging, these techniques could enable precise determination of the intracellular site of seeding activity.

Non-FRET-based biosensor cells also detect aggregation. Complementation assays are based on splitting a functional protein, such as GFP into two halves, with each individually fused to a target protein. On aggregation of the target protein, the GFP halves come together to restore activity (Cabantous et al. 2005). Alternatively, a holoprotein may lose activity on aggregation. Biosensors that "turn off" on aggregation (Chun and Johnson 2007; Chun et al. 2007) and those that "turn on" (Tak et al. 2013; Kim et al. 2015) permit the dynamic assessment of tau aggregation under different phosphorylation states. We have recently published the use of a split luciferase complementation assay in 
which tau aggregation leads to functional reconstitution of the enzyme and emits light in the presence of luciferin. This assay requires minimal experimental setup and can be conducted on any luminescence plate reader. Although this method does not provide spatial resolution for intracellular tau aggregation (in contrast to the GFP variants of the assay), it has broad dynamic range and high sensitivity. Using this approach, we established that tau trimers are the minimum unit of infectivity in cells, using both recombinant and $\mathrm{AD}$-derived tau (Mirbaha et al. 2015).

Finally, Guo et al. (2013) used standard immunocytochemistry with antibodies that recognize pathological forms of tau to detect seeding. This approach has been used in conjunction with high content microscopy (Usenovic et al. 2015). All the assays described can be established for primary neurons, in high throughput, and in automated formats. These platforms will allow for systematic screening to identify novel molecular targets to block transcellular propagation of proteopathic seeds.

\section{TRANSCELLULAR PROPAGATION}

The transcellular propagation of tau aggregation through neural networks may underlie the stereotyped and hierarchical progression of neuropathology in diseased brains. Animal inoculation studies have shown that tau pathology spreads from brain region to region determined by synaptic connectivity, not proximity (de Calignon et al. 2012; Liu et al. 2012; Ahmed et al. 2014). Thus, the synapse may be the critical cellular site of propagation through networks, requiring unique experimental paradigms for cellular studies.

Calafate et al. (2015) recently developed a cell culture system to interrogate the role of synapses in tau seed propagation. They co-cultured a monoclonal HEK293 cell line that stably produces tau aggregates (donor cells) with primary hippocampal neurons expressing human P301L tau (acceptor cells). Using a combination of microscopy and biochemistry, they detected pathological tau in the neuronal acceptor cells when they were co-cultured with seed-containing donor cells, but not with control cells expressing monomeric, soluble tau. This suggested that tau pathology propagated from the monoclonal cell donor to the primary neuron acceptor. Notably, they also observed that synaptic connections enhance tau seed propagation. Expression of synaptogenic proteins Nlg1 and LRRTM4 leads to the de novo formation of presynaptic structures in non-neuronal cells (Scheiffele et al. 2000; de Wit et al. 2009). By using this technique, Calafate et al. (2015) created artificial synapses between the donor and acceptor neurons, which increased seeded pathology by $50 \%$ in the acceptors. This effect was not mediated simply by increased cell contact because overexpression of $\mathrm{N}$-cadherin, which leads to increased cell:neuron contact but no changes in the number of synapses, did not alter the propagation of tau pathology.

Finally, Calafate et al. (2015) directly tested the role of synapses in a neuron-to-neuron culture system, exploiting three isolated microfluidic chambers separated by microgrooves. Neurons within each chamber extend axons (but not dendrites) via the microgrooves to form synaptic connections with neurons in the adjacent chamber. Hydrostatic gradients preclude diffusion through the chambers, and, thus, passage of material from one compartment to the next can only occur via synaptic connections. Using this platform, Calafate et al. (2015) seeded aggregation in the first neuronal chamber. Over the ensuing 2 weeks, tau accumulated in neurons in the second and third chambers, consistent with transcellular propagation of tau aggregates. Reduction of synapses via knockdown of neuroligins or inhibition of synaptic activity by tetrodotoxin reduced neuron-to-neuron propagation of tau pathology (Calafate et al. 2015). Taken together, these data are consistent with the idea that both nonsynaptic and synaptic mechanisms could contribute to the propagation of tau pathology, but that synaptic connections enhance the process. With these tools, it should be feasible to identify the molecular mechanisms of trans-synaptic propagation. It remains unknown whether trans-synaptic tau movement is guided by neurotransmission 
itself or whether the synaptic architecture merely provides a more efficient path for propagation because of the close juxtaposition of cell membranes.

Other laboratories have also exploited microfluidic compartments to study tau seed transmission (Freundt et al. 2012; Wu et al. 2012; Tran et al. 2014; Takeda et al. 2015). When evaluating the results from these studies, it is important to keep in mind whether the cell assay reports on true propagation of tau aggregation (i.e., serial induction of de novo aggregation from each cell's endogenous tau) (Tran et al. 2014; Calafate et al. 2015), or whether the primary inoculated seed merely transfers from cell to cell without further induction of aggregation (Freundt et al. 2012; Takeda et al. 2015). True propagation of tau proteopathic seeding is likely required to drive neurodegeneration and to amplify and maintain infectious seed titers throughout the brain. Nonetheless, these assays will help establish critical features of tau aggregate transmission that may provide mechanistic clues for drug targets.

\section{PRION STRAINS}

Prion diseases show phenotypic diversity, including variation in incubation time and patterns of neuropathology, which can be explained by distinct biophysical properties of $\mathrm{PrP}^{\mathrm{Sc}}$ (Collinge et al. 1996; Telling et al. 1996). $\mathrm{PrP}^{\mathrm{Sc}}$ strains have several critical characteristics: they are linked to defined amyloid structures, maintained faithfully in serial passage in living systems, and produce predictable and well-defined strain-specific disease and neuropathology. In contrast, amyloid preparations that simply have distinct conformations in vitro (which may or may not produce different pathologies on inoculation in vivo), or consist of mixed conformational assemblies, are not bona fide strains unless they stably propagate these characteristics in living systems. Experimental data strongly support the idea that unique conformational states, or strains, of the $\operatorname{PrP}^{\mathrm{Sc}}$ amyloid underlie the myriad prion-disease clinical syndromes (e.g., Bartz 2016; Ghaemmaghami 2016.)
In recent years, the strain concept has been extended to common amyloid disorders. First, neurodegenerative diseases, such as tauopathies and synucleinopathies show great phenotypic diversity that could, in theory, arise from distinct protein conformations (Lee et al. 2001; Frost et al. 2009b; McCann et al. 2014). For example, AD, corticobasal degeneration, progressive supranuclear palsy, Pick's disease, argyrophilic grain disease, and other tauopathies have distinct clinical and neuropathological presentations (Lee et al. 2001). Second, many proteins, in addition to $\operatorname{PrP}^{\mathrm{Sc}}$, show polymorphic amyloid states, including $A \beta$, tau, and $\alpha$-synuclein (Petkova et al. 2005; Frost et al. 2009b; Bousset et al. 2013; Guo et al. 2013; Lu et al. 2013). Distinct strains could, thus, give rise to diverse neurodegenerative syndromes associated with protein amyloids.

In 2014, our laboratory provided definitive cellular and molecular evidence for tau strains by establishing a cell culture system that indefinitely propagates unique tau conformations with high fidelity. We stably transduced HEK293 cells with a mutant form of tau repeat domain fused to YFP and triggered intracellular aggregation with exogenous recombinant tau seeds. We isolated monoclonal colonies consisting of two unique strains based on distinct inclusion morphologies and biochemical and structural characteristics of the aggregated tau. Stable mother-to-daughter cell transmission occurred during cell division over months in culture. We then inoculated these strains into the P301S tauopathy mouse model. This induced unique neuronal tau pathology that we observed consistently on serial passage. After three generations in mice, tau strains maintained their unique conformations when introduced into the original naïve reporter cell lines (Sanders et al. 2014). Thus, like $\operatorname{PrP}^{\mathrm{Sc}}$, tau amyloids form distinct and self-perpetuating prion strains.

We next used cell models to test whether human tauopathies are linked to unique tau conformations. Inoculation of brain lysate from five different tauopathy syndromes into the cell reporter system generated multiple disease-associated tau strain patterns (Sanders 
et al. 2014). These results complemented previous findings that human brain homogenates from different tauopathy syndromes also produce distinct patterns of neuropathology on inoculation into mice (Clavaguera et al. 2013). The use of a simple cell reporter system has, thus, permitted the facile production and isolation of unique tau strains. Although only tau prion strains have been definitively described, the principles of strain propagation will likely extend to multiple amyloid diseases.

\section{CONCLUSION}

The last decade has brought enormous growth in our understanding of prion biology's relationship to common neurodegenerative diseases. Over the past 30 years, the study of PrPassociated diseases has elucidated principles that now contribute to our understanding of common disorders, such as Alzheimer's, Parkinson's, and the motor neuron diseases. Mouse models have been the mainstay of biological studies of $\operatorname{PrP}^{\mathrm{Sc}}$ infectivity and strain analyses. Now, however, by exploiting molecular biology, it is straightforward to engineer cellular models to study the prion biology of proteins such as tau and $\alpha$-synuclein. These models allow quantitative assessment of multiple facets of prion disease, including cell binding, uptake, seeding, transcellular propagation, and strain characteristics. Initial observations focused on binding and uptake of amyloidogenic proteins in cultured cells. In the case of tau and $\alpha$-synuclein, this enabled the elucidation of the molecular mechanism of cell uptake: heparan sulfate proteoglycans and macropinocytosis. In addition to animal inoculation studies, biosensor cell models now also permit assessment of tau and $\alpha$-synuclein seeding activity in brain samples. In turn, studies with cellular models have strongly suggested that seeding activity precedes and predicts subsequent neuropathology. In conjunction with high content microscopy and real-time imaging, cellular models also promise to elucidate the subcellular localization of seed amplification and its mechanisms. Likewise, transcellular propagation, which previously was very difficult to study in vivo, is now a more tractable problem with newer cellular models. These exploit microfluidic culture chambers and have enabled researchers to track aggregate movement across synapses and the spread of seeding activity from one cell to another. Finally, cell models have allowed better characterization of prion strains, which are distinct amyloid conformations that lead to predictable types of neuropathology. It is now clear that tau prion strains propagate from mother to daughter cells. Clonal cell lines have enabled propagation of discrete strains, enabling their isolation and precise characterization. In the future, we anticipate that cellular models of prion biology, when combined with cutting-edge genetic technologies, will underlie enormous progress in a mechanistic understanding of neurodegenerative diseases.

\section{REFERENCES}

${ }^{*}$ Reference is also in this collection.

Ahmed Z, Cooper J, Murray TK, Garn K, McNaughton E, Clarke H, Parhizkar S, Ward MA, Cavallini A, Jackson S, et al. 2014. A novel in vivo model of tau propagation with rapid and progressive neurofibrillary tangle pathology: The pattern of spread is determined by connectivity, not proximity. Acta Neuropathol 127: 667-683.

* Bartz JC. 2016. Prion strain diversity. Cold Spring Harb Perspect Med doi: 10.1101/cshperspect.a024349.

Bellinger-Kawahara CG, Kempner E, Groth D, Gabizon R, Prusiner SB. 1988. Scrapie prion liposomes and rods exhibit target sizes of 55,000 Da. Virology 164: 537-541.

Bousset L, Pieri L, Ruiz-Arlandis G, Gath J, Jensen PH, Habenstein B, Madiona K, Olieric V, Bockmann A, Meier BH, et al. 2013. Structural and functional characterization of two $\alpha$-synuclein strains. Nat Commun 4: 2575 .

Cabantous S, Terwilliger TC, Waldo GS. 2005. Protein tagging and detection with engineered self-assembling fragments of green fluorescent protein. Nat Biotechnol 23: $102-107$.

Calafate S, Buist A, Miskiewicz K, Vijayan V, Daneels G, de Strooper B, de Wit J, Verstreken P, Moechars D. 2015. Synaptic contacts enhance cell-to-cell tau pathology propagation. Cell Rep 11: 1176-1183.

Chun W, Johnson GV. 2007. Activation of glycogen synthase kinase $3 \beta$ promotes the intermolecular association of tau. The use of fluorescence resonance energy transfer microscopy. J Biol Chem 282: 23410-23417.

Chun W, Waldo GS, Johnson GV. 2007. Split GFP complementation assay: A novel approach to quantitatively measure aggregation of tau in situ: Effects of GSK3 $\beta$ activation and caspase 3 cleavage. J Neurochem 103: 25292539. 
Clavaguera F, Bolmont T, Crowther RA, Abramowski D, Frank S, Probst A, Fraser G, Stalder AK, Beibel M, Staufenbiel M, et al. 2009. Transmission and spreading of tauopathy in transgenic mouse brain. Nat Cell Biol 11: 909-913.

Clavaguera F, Akatsu H, Fraser G, Crowther RA, Frank S, Hench J, Probst A, Winkler DT, Reichwald J, Staufenbiel M, et al. 2013. Brain homogenates from human tauopathies induce tau inclusions in mouse brain. Proc Natl Acad Sci 110: 9535-9540.

* Clavaguera F, Tolnay M, Goedert M. 2016. The prion-like behavior of assembled tau in transgenic mice. Cold Spring Harb Perspect Med doi: 10.1101/cshperspect.a024372.

Collinge J, Sidle KC, Meads J, Ironside J, Hill AF. 1996. Molecular analysis of prion strain variation and the aetiology of "new variant" CJD. Nature 383: 685-690.

de Calignon A, Polydoro M, Suárez-Calvet M, William C, Adamowicz DH, Kopeikina KJ, Pitstick R, Sahara N, Ashe KH, Carlson GA, et al. 2012. Propagation of tau pathology in a model of early Alzheimer's disease. Neuron 73: 685-697.

de Wit J, Sylwestrak E, O'Sullivan ML, Otto S, Tiglio K, Savas JN, Yates JR III, Comoletti D, Taylor P, Ghosh A. 2009. LRRTM2 interacts with Neurexin1 and regulates excitatory synapse formation. Neuron 64: 799-806.

Freundt EC, Maynard N, Clancy EK, Roy S, Bousset L, Sourigues Y, Covert M, Melki R, Kirkegaard K, Brahic M. 2012. Neuron-to-neuron transmission of $\alpha$-synuclein fibrils through axonal transport. Ann Neurol 72: 517524.

Frost B, Jacks RL, Diamond MI. 2009a. Propagation of tau misfolding from the outside to the inside of a cell. J Biol Chem 284: 12845-12852.

Frost B, Ollesch J, Wille H, Diamond MI. 2009b. Conformational diversity of wild-type tau fibrils specified by templated conformation change. J Biol Chem 284: 3546-3551.

Funk KE, Mirbaha H, Jiang H, Holtzman DM, Diamond MI. 2015. Distinct therapeutic mechanisms of tau antibodies: Promoting microglial clearance versus blocking neuronal uptake. J Biol Chem 290: 21652-21662.

* Ghaemmaghami S. 2016. Biology and genetics of PrP prion strains. Cold Spring Harb Perspect Med doi: 10.1101/ cshperspect.a026922.

Guo JL, Covell DJ, Daniels JP, Iba M, Stieber A, Zhang B, Riddle DM, Kwong LK, Xu Y, Trojanowski JQ, et al. 2013. Distinct $\alpha$-synuclein strains differentially promote tau inclusions in neurons. Cell 154: 103-117.

Holmes BB, DeVos SL, Kfoury N, Li M, Jacks R, Yanamandra K, Ouidja MO, Brodsky FM, Marasa J, Bagchi DP, et al. 2013. Heparan sulfate proteoglycans mediate internalization and propagation of specific proteopathic seeds. Proc Natl Acad Sci 110: E3138-E3147.

Holmes BB, Furman JL, Mahan TE, Yamasaki TR, Mirbaha H, Eades WC, Belaygorod L, Cairns NJ, Holtzman DM, Diamond MI. 2014. Proteopathic tau seeding predicts tauopathy in vivo. Proc Natl Acad Sci 111: E4376-E4385.

Horonchik L, Tzaban S, Ben-Zaken O, Yedidia Y, Rouvinsk A, Papy-Garcia D, Barritault D, Vlodavsky I, Taraboulos A. 2005. Heparan sulfate is a cellular receptor for purified infectious prions. J Biol Chem 280: 17062-17067.
Studying Prion Biology in Cultured Cells

Kaminski Schierle GS, Bertoncini CW, Chan FT, van der Goot AT, Schwedler S, Skepper J, Schlachter S, van Ham T, Esposito A, Kumita JR, et al. 2011. A FRET sensor for non-invasive imaging of amyloid formation in vivo. Chemphyschem 12: 673-680.

Kaufman SK, Sanders DW, Talitha L, Thomas TL. Neuron (in press).

Kfoury N, Holmes BB, Jiang H, Holtzman DM, Diamond MI. 2012. Trans-cellular propagation of tau aggregation by fibrillar species. J Biol Chem 287: 19440-19451.

Kim D, Lim S, Haque MM, Ryoo N, Hong HS, Rhim H, Lee DE, Chang YT, Lee JS, Cheong E, et al. 2015. Identification of disulfide cross-linked tau dimer responsible for tau propagation. Sci Rep 5: 15231.

Lee VM, Goedert M, Trojanowski JQ. 2001. Neurodegenerative tauopathies. Annu Rev Neurosci 24: 1121-1159.

Lee HJ, Suk JE, Bae EJ, Lee JH, Paik SR, Lee SJ. 2008. Assembly-dependent endocytosis and clearance of extracellular $\alpha$-synuclein. Int J Biochem Cell Biol 40: 1835-1849.

Liu L, Drouet V, Wu JW, Witter MP, Small SA, Clelland C, Duff K. 2012. Trans-synaptic spread of tau pathology in vivo. PLoS ONE 7: e31302.

Lu JX, Qiang W, Yau WM, Schwieters CD, Meredith SC, Tycko R. 2013. Molecular structure of $\beta$-amyloid fibrils in Alzheimer's disease brain tissue. Cell 154: 1257-1268.

McCann H, Stevens CH, Cartwright H, Halliday GM. 2014. $\alpha$-Synucleinopathy phenotypes. Parkinsonism Relat Disord 20: S62-S67.

Michel CH, Kumar S, Pinotsi D, Tunnacliffe A, St GeorgeHyslop P, Mandelkow E, Mandelkow EM, Kaminski CF, Kaminski Schierle GS. 2013. Extracellular monomeric tau is sufficient to initiate the spread of tau pathology. $J$ Biol Chem 289: 956-967.

Mirbaha H, Holmes BB, Sanders DW, Bieschke J, Diamond MI. 2015. Tau trimers are the minimal propagation unit spontaneously internalized to seed intracellular aggregation. J Biol Chem 290: 14893-14903.

Petkova AT, Leapman RD, Guo Z, Yau WM, Mattson MP, Tycko R. 2005. Self-propagating, molecular-level polymorphism in Alzheimer's $\beta$-amyloid fibrils. Science 307: 262-265.

Pollitt SK, Pallos J, Shao J, Desai UA, Ma AA, Thompson LM, Marsh JL, Diamond MI. 2003. A rapid cellular FRET assay of polyglutamine aggregation identifies a novel inhibitor. Neuron 40: 685-694.

Prusiner SB. 1998. Prions. Proc Natl Acad Sci 95: $13363-$ 13383.

Sanders DW, Kaufman SK, DeVos SL, Sharma AM, Mirbaha H, Li A, Barker SJ, Foley AC, Thorpe JR, Serpell LC, et al. 2014. Distinct tau prion strains propagate in cells and mice and define different tauopathies. Neuron 82: 1271-1288.

Scheiffele P, Fan J, Choih J, Fetter R, Serafini T. 2000. Neuroligin expressed in nonneuronal cells triggers presynaptic development in contacting axons. Cell 101: 657-669.

Tak H, Haque MM, Kim MJ, Lee JH, Baik JH, Kim Y, Kim DJ, Grailhe R, Kim YK. 2013. Bimolecular fluorescence complementation; lighting-up tau-tau interaction in living cells. PLoS ONE 8: e81682.

Takeda S, Wegmann S, Cho H, DeVos SL, Commins C, Roe AD, Nicholls SB, Carlson GA, Pitstick R, Nobuhara CK, 
B.B. Holmes and M.I. Diamond

et al. 2015. Neuronal uptake and propagation of a rare phosphorylated high-molecular-weight tau derived from Alzheimer's disease brain. Nat Commun 6: 8490 .

Taylor AM, Blurton-Jones M, Rhee SW, Cribbs DH, Cotman CW, Jeon NL. 2005. A microfluidic culture platform for CNS axonal injury, regeneration and transport. Nat Methods 2: 599-605.

Telling GC, Parchi P, DeArmond SJ, Cortelli P, Montagna P, Gabizon R, Mastrianni J, Lugaresi E, Gambetti P, Prusiner SB. 1996. Evidence for the conformation of the pathologic isoform of the prion protein enciphering and propagating prion diversity. Science 274: 2079-2082.

Tran HT, Chung CH, Iba M, Zhang B, Trojanowski JQ, Luk KC, Lee VM. 2014. $\alpha$-Synuclein immunotherapy blocks uptake and templated propagation of misfolded $\alpha$-synuclein and neurodegeneration. Cell Rep 7: 2054-2065.
Usenovic M, Niroomand S, Drolet RE, Yao L, Gaspar RC, Hatcher NG, Schachter J, Renger JJ, Parmentier-Batteur S. 2015. Internalized tau oligomers cause neurodegeneration by inducing accumulation of pathogenic tau in human neurons derived from induced pluripotent stem cells. J Neurosci 35: 14234-14250.

Wu JW, Herman M, Liu L, Simoes S, Acker CM, Figueroa H, Steinberg JI, Margittai M, Kayed R, Zurzolo C, et al. 2012. Small misfolded tau species are internalized via bulk endocytosis and anterogradely and retrogradely transported in neurons. J Biol Chem 288: 18561870.

Yanamandra K, Kfoury N, Jiang H, Mahan TE, Ma S, Maloney SE, Wozniak DF, Diamond MI, Holtzman DM. 2013. Anti-tau antibodies that block tau aggregate seeding in vitro markedly decrease pathology and improve cognition in vivo. Neuron 80: 402-414. 


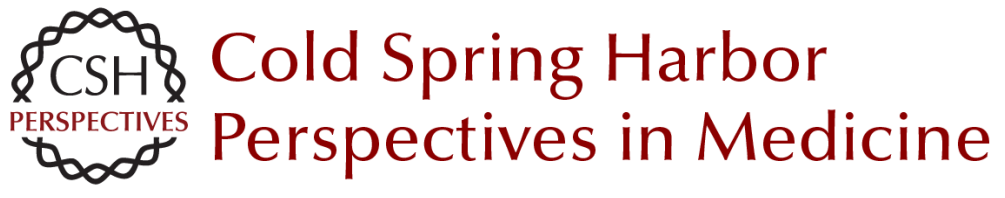

\section{Cellular Models for the Study of Prions}

Brandon B. Holmes and Marc I. Diamond

Cold Spring Harb Perspect Med 2017; doi: 10.1101/cshperspect.a024026 originally published online November 4, 2016

\section{Subject Collection Prion Diseases}

TDP-43 Prions

Takashi Nonaka and Masato Hasegawa

$\alpha$-Synuclein: Multiple System Atrophy Prions Amanda L. Woerman, Joel C. Watts, Atsushi Aoyagi, et al.

Genetics of Synucleinopathies Robert L. Nussbaum

$\beta$-Amyloid Prions and the Pathobiology of

Alzheimer's Disease Joel C. Watts and Stanley B. Prusiner

Disease Mechanisms of C9ORF72 Repeat Expansions

Tania F. Gendron and Leonard Petrucelli

Chronic Traumatic Encephalopathy: Is Latency in

Symptom Onset Explained by Tau Propagation? Joshua Kriegel, Zachary Papadopoulos and Ann C. McKee

Noncerebral Amyloidoses: Aspects on Seeding,

Cross-Seeding, and Transmission

Gunilla T. Westermark, Marcus Fändrich,

Katarzyna Lundmark, et al.

Structural and Chemical Biology of Presenilin

Complexes

Douglas S. Johnson, Yue-Ming Li, Martin

Pettersson, et al.
Cell Biology and Pathophysiology of $\alpha$-Synuclein Jacqueline Burré, Manu Sharma and Thomas C. Südhof

Molecular Mechanisms of Chronic Wasting

Disease Prion Propagation Julie A. Moreno and Glenn C. Telling

Genetics of Amyotrophic Lateral Sclerosis Mehdi Ghasemi and Robert H. Brown, Jr.

The Genetics of C9orf72 Expansions Ilse Gijselinck, Marc Cruts and Christine Van Broeckhoven

Prion-Like Characteristics of

Polyglutamine-Containing Proteins Margaret M.P. Pearce and Ron R. Kopito

Therapeutic Strategies for Restoring Tau Homeostasis

Zapporah T. Young, Sue Ann Mok and Jason E. Gestwicki

Fused in Sarcoma Neuropathology in Neurodegenerative Disease Ian R.A. Mackenzie and Manuela Neumann

Experimental Models of Inherited PrP Prion Diseases Joel C. Watts and Stanley B. Prusiner

For additional articles in this collection, see http://perspectivesinmedicine.cshlp.org/cgi/collection/ 